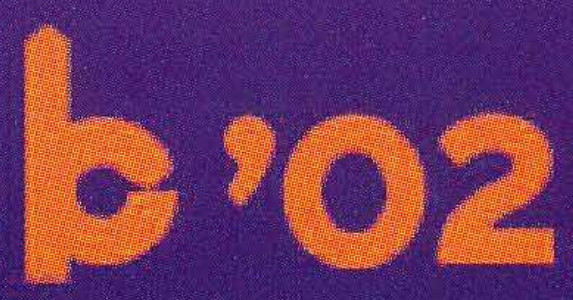

Preprints of the

15th TRIENNIAL WORLD CONGRESS of the

INTERNATIONAL FEDERATION OF AUTOM ATIC CONTROL
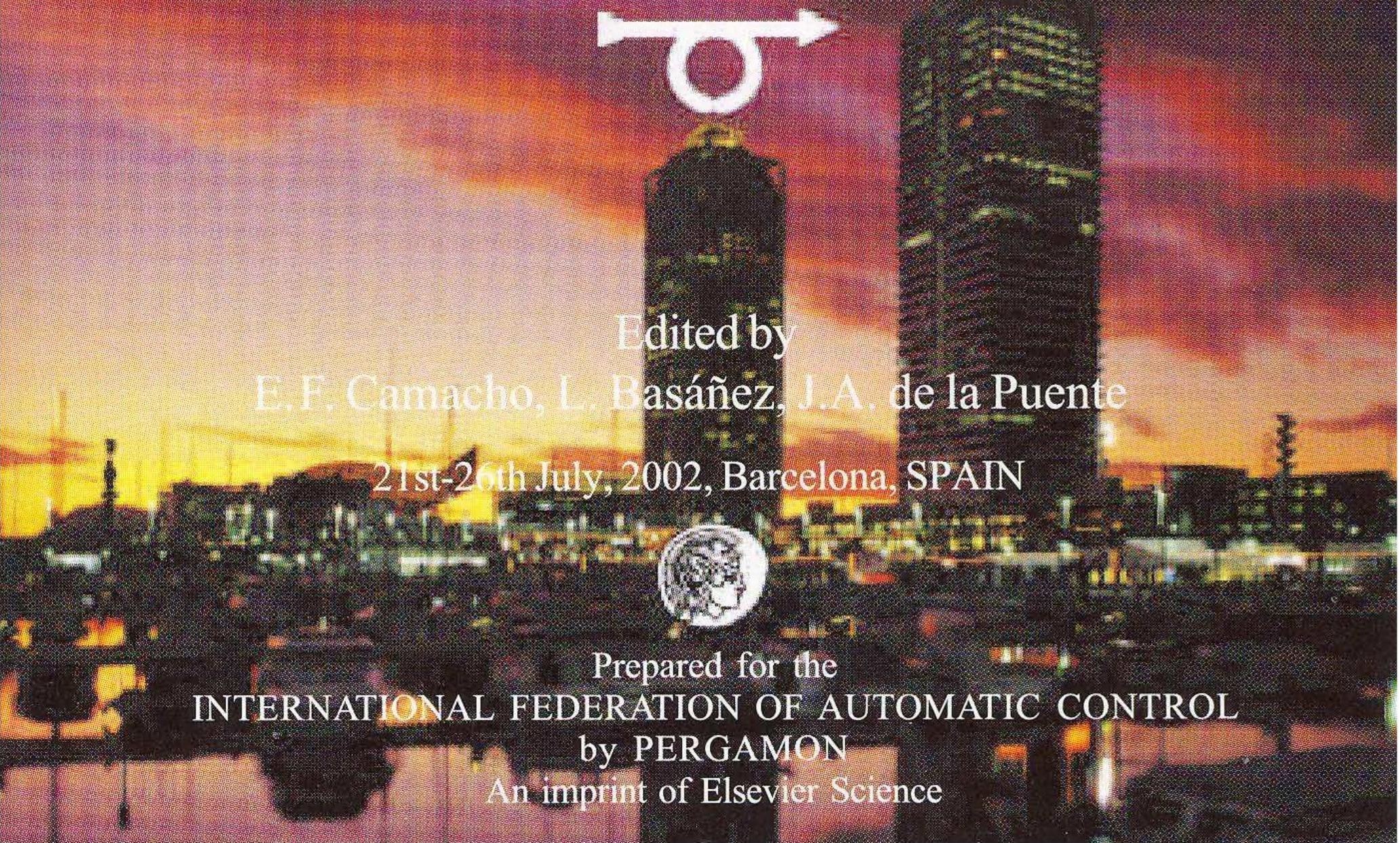



\title{
POWER ANALYSIS OF MULTI-LEGGED SYSTEMS
}

\author{
Manuel F. Silva *, J. A. Tenreiro Machado *, António M. Lopes ${ }^{+}$ \\ * Dept. of Electrical Engineering, Institute of Engineering of Porto, \\ Rua Dr. António Bernardino de Almeida, 4200-072 Porto, Portugal \\ Email: $\{m f s, j t m\} @ d e e . i s e p . i p p . p t$ \\ ${ }^{+}$Dept. of Mechanical Engineering, Faculty of Engineering of Porto \\ Rua Dr. Roberto Frias, 4200-465 Porto, Portugal \\ Email:aml@fe.up.pt
}

\begin{abstract}
This paper studies periodic gaits of multi-legged robot locomotion systems based on dynamic models. The purpose is to determine the system performance during walking and the best set of locomotion variables that minimizes the optimization indices. For that objective the prescribed motion of the robot is completely characterized in terms of several locomotion variables such as gait, duty factor, body height, step length, stroke pitch, foot clearance, leg links length, foot-hip offset, body and legs mass and cycle time. In this perspective, we formulate four performance measures of the walking robot namely, the foot locomobility index, the mean absolute power, the mean power dispersion and the mean power lost in the joint actuators per walking distance. A set of model-based experiments reveals the influence of the locomotion variables in the proposed indices. Copyright (C) 2002 IFAC
\end{abstract}

Keywords - legged locomotion, robotics, simulation

\section{INTRODUCTION}

Walking machines allow locomotion in terrain inaccessible to other type of vehicles, since they do not need a continuous support surface (Manko, 1992; Song and Waldron, 1989). On the other hand, the requirements for leg coordination and control impose difficulties beyond those encountered in wheeled robots (Caux and Zapata, 1997; Nelson and Quinn, 1999; Pfeiffer et al., 1995). Gait selection is a research area requiring an appreciable modeling effort for the improvement of mobility with legs in unstructured environments (Jimenez and Santos, 1997; Wettergreen and Thorpe, 1992). Previous studies focused in the structure and selection of locomotion modes (Venkataraman, 1996; Wettergreen et al., 1995). Nevertheless, there are different optimization criteria such as energy efficiency, stability, velocity, comfort, mobility and environmental impact (Gregorio et al., 1997; Lapshin, 1995; Zielinska, 2000). With these facts in mind, a simulation model for multi-leg locomotion systems was developed, for several periodic gaits (Song and Waldron, 1989). This study intends to generalize previous work (Silva et al., 2001a; Silva et al., 2001b; Silva and Machado, 1999) through the formulation of several indices measuring the system locomobility, the average power during different walking trajectories, the standard deviation of the power consumption and the power lost in the joint actuators along the space-time walking cycle.

The foot and body trajectories are analyzed in what concerns its variation with the gait, duty factor, step length, maximum foot clearance, body height, leg links length and foot-hip offset. Several simulation experiments reveal the system configuration and the type of the movements that lead to a better mechanical implementation, for a given locomotion mode, from the viewpoint of the proposed indices.

Bearing these facts in mind, the paper is organized as follows. Section two introduces the model for a multi-legged robot and the motion planning algorithms. Section three formulates the optimizing indices and section four develops a set of experiments that reveal the influence of the system parameters in the periodic gaits, respectively. Finally, section five presents the main conclusions and directions towards future developments. 


\section{A MODEL FOR MULTI-LEGGED LOCOMOTION}

We consider a longitudinal walking system with $n$ legs ( $n \geq 2$ and $n$ even), with the legs equally distributed along both sides of the robot body, having each one two rotational joints (Fig. 1).

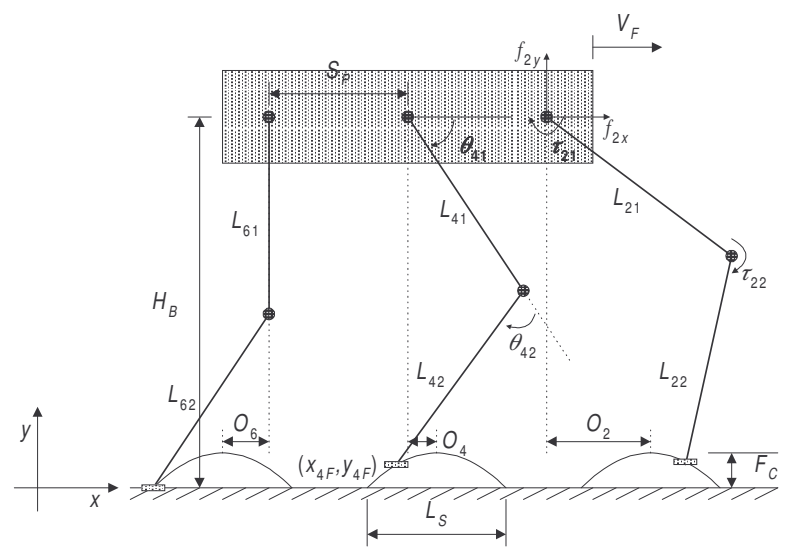

Fig. 1. Coordinate system and variables that characterize the motion trajectories of the multilegged robot

Motion is described by means of a world coordinate system. Defining the cycle time $T$, the duty factor $\beta$, the transference time $t_{T}=(1-\beta) T$, the support time $t_{S}=\beta T$, the step length $L_{S}$, the stroke pitch $S_{P}$, the body height $H_{B}$, the maximum foot clearance $F_{C}$, the $i^{\text {th }}$ leg lengths $L_{i 1}$ and $L_{i 2}$ and the foot trajectory offset $O_{i}(i=1, \ldots, n)$ we consider a periodic trajectory for each foot, with body velocity $V_{F}=L_{S} / T$.

The algorithm for the forward motion planning accepts the body and $i^{\text {th }}$ feet cartesian trajectories $\mathbf{p}_{\mathbf{F}}(t)=\left[x_{i F}(t), y_{i F}(t)\right]^{\mathrm{T}}$ as inputs and, by means of an inverse kinematics algorithm, generates the related joint trajectories $\boldsymbol{\theta}(t)=\left[\theta_{i 1}(t), \theta_{i 2}(t)\right]^{\mathrm{T}}$, selecting the solution corresponding to a forward knee.

The body of the robot, and by consequence the legs hips, are assumed to have a horizontal movement with a constant forward speed $V_{F}$. Therefore, for leg $i$ the cartesian coordinates of the hip of the legs are given by:

$$
\mathbf{p}_{\mathbf{H}}(t)=\left[\begin{array}{c}
x_{i H}(t) \\
y_{i H}(t)
\end{array}\right]=\left[\begin{array}{c}
V_{F} \cdot t \\
H_{B}
\end{array}\right]
$$

Given a particular gait and duty factor $\beta$, it is possible (Song and Waldron, 1989) to calculate for leg $i$ the corresponding phase $\phi_{i}$ and the time instant where each leg leaves and returns to contact with the ground. From these results, and knowing $T, \beta$ and $t_{S}$, the cartesian trajectories of the tip of the foots must be completed during $t_{T}$.

For each cycle the trajectory of the tip of the swing leg is computed through a cycloid function given by (considering, for example, that the transfer phase starts at $t=0 \sec$ for leg 1 ), with $f=1 / T$ :

- during the transfer phase:

$$
\begin{gathered}
x_{1 F}(t)=V_{F}\left[t-\frac{1}{2 \pi f} \sin (2 \pi f t)\right] \\
y_{1 F}(t)=\frac{F_{C}}{2}[1-\cos (2 \pi f t)]
\end{gathered}
$$

- during the stance phase:

$$
\begin{gathered}
x_{1 F}(t)=V_{F}\left[T-\frac{1}{2 \pi f} \sin (2 \pi f T)\right]=V_{F} T \\
y_{1 F}(t)=0
\end{gathered}
$$

From the coordinates of the hip and feet of the robot it is possible to obtain the leg joint positions and velocities using the inverse kinematics:

$$
\begin{gathered}
\mathbf{p}(t)=\left[\begin{array}{l}
x_{i}(t) \\
y_{i}(t)
\end{array}\right]=\mathbf{p}_{\mathbf{H}}(t)-\mathbf{p}_{\mathbf{F}}(t) \\
\boldsymbol{\theta}(t)=f[\mathbf{p}(t)] \\
\dot{\boldsymbol{\theta}}(t)=\mathbf{J}^{-1}[\dot{\mathbf{p}}(t)]
\end{gathered}
$$

Based on this data, the trajectory generator is responsible for producing a motion that synchronises and co-ordinates the legs. In order to avoid the impact and friction effects we impose null velocities of the feet in the instants of landing and taking off, assuring also the velocity continuity. These joint trajectories can also be accomplished either with a step or a polynomial versus time acceleration profile. After planning the joint trajectories we calculate the inverse dynamics in order to 'map' the kinematics into power consumption. The robot inverse dynamic model is of the form:

$$
\boldsymbol{\tau}=\mathbf{H}(\boldsymbol{\theta}) \ddot{\boldsymbol{\theta}}+\mathbf{c}(\boldsymbol{\theta}, \dot{\boldsymbol{\theta}})+\mathbf{g}(\boldsymbol{\theta})
$$

where $\tau=\left[f_{i x}, f_{i y}, \tau_{i 1}, \tau_{i 2}\right]^{\mathrm{T}}(i=1, \ldots, n)$ is the vector of forces/torques, $\boldsymbol{\theta}=\left[x_{i}, y_{i}, \theta_{i 1}, \theta_{i 2}\right]^{\mathrm{T}}$ is the vector of position coordinates, $\mathbf{H}(\boldsymbol{\theta})$ is the inertia matrix and $\mathbf{c}(\boldsymbol{\theta}, \dot{\boldsymbol{\theta}})$ and $\mathbf{g}(\boldsymbol{\theta})$ are the vectors of centrifugal/Coriolis and gravitational forces/torques, respectively.

\section{MEASURES FOR PERFORMANCE EVALUATION}

In mathematical terms, we provide four global measures of the overall performance of the mechanism in an average sense.

\subsection{Locomobility Measure}

The motivation for the development of the locomobility index is to apply the concepts of arm manipulability to multi-legged walking (Yoshikawa, 1990). This performance measure can be expressed through the Jacobian matrix. In our case, the global index is obtained by averaging the distance among the centre of the ellipsoids and its intersections with the tangent to the desired trajectories of the foot, at the centre of the ellipsoid, $\left(E_{F}\right)$, over a complete cycle $T$ (Fig. 2): 


$$
L_{F}=\frac{1}{T} \int_{0}^{T} E_{F}(t) d t
$$

In this perspective, the most suitable trajectory is the one that maximises $L_{F}$.

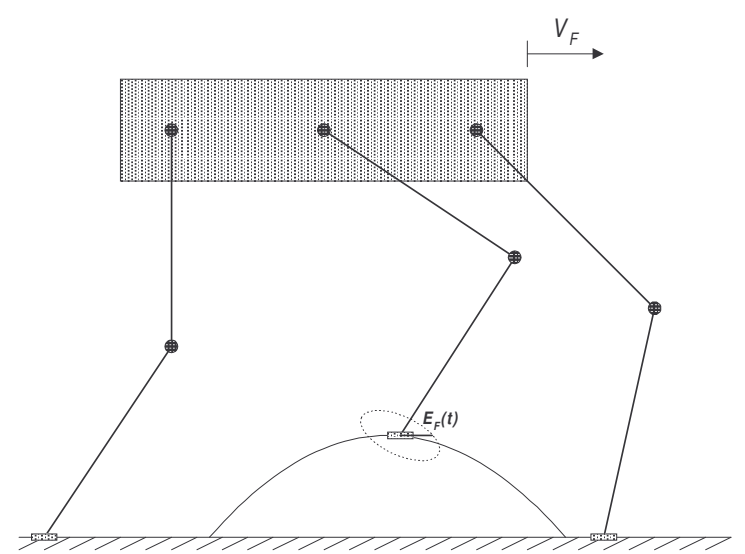

Fig. 2. Graphical representation of the distance among the centre of the ellipsoids and its intersections with the tangent to the trajectory of the foot $\left(E_{F}\right)$

\subsection{Mean Absolute Power}

The key measure in this analysis is the mean absolute power per travelling distance. It is computed assuming that power regeneration is not available by actuators doing negative work, that is, by taking the absolute value of the power. At a given joint $j$ (each leg has $m=2$ joints) and leg $i$ (since we are adopting an hexapod it yields $n=6$ legs), the mechanical power is the product of the motor torque and angular velocity. The global index is obtained by averaging the mechanical absolute power delivered over a period $T$ and a step $L_{S}$ :

$$
P_{a v}=\frac{1}{L_{S}} \cdot \frac{1}{T} \sum_{i=1}^{n} \sum_{j=1}^{m} \int_{0}^{T}\left|\tau_{i j}(t) \cdot \dot{\theta}_{i j}(t)\right| d t
$$

The average of the absolute power consumption per travelling distance, $P_{a v}$, should be minimised.

\subsection{Mean Power Dispersion}

Although minimising power appears to be an important consideration, it may occur instantaneous, very high, power demands. In such cases, the average value can be small while the peaks are physically unrealisable. An alternative index is the standard deviation per meter that evaluates the dispersion around the mean absolute power over a complete cycle $T$ and step length:

$$
\begin{gathered}
P_{i}(t)=\sum_{i=1}^{n} \sum_{j=1}^{m}\left|\boldsymbol{\tau}_{i j}(t) \cdot \dot{\boldsymbol{\theta}}_{i j}(t)\right| \\
D_{a v}=\frac{1}{L_{S}} \cdot \sqrt{\frac{1}{T} \int_{0}^{T}\left[P_{i}(t)-P_{a v}\right]^{2} d t}
\end{gathered}
$$

where $P_{i}$ is the total instantaneous absolute mechanical power. In this line of thought, the most suitable trajectory is the one that minimizes $D_{a v}$.

\subsection{Mean Power Lost}

Another optimisation strategy for an actuated system considers the power lost in the joint actuators per cycle $T$ and step length $L_{S}$. From this point of view, the index mean power lost per meter can be defined as:

$$
P_{L}=\frac{1}{L_{S}} \cdot \frac{1}{T} \sum_{i=1}^{n} \sum_{j=1}^{m} \int_{0}^{T}\left[\tau_{i j}(t)\right]^{2} d t
$$

The most suitable trajectory is the one that minimizes $P_{L}$.

\section{SIMULATION RESULTS}

To illustrate the use of the preceding concepts, in this section we develop a set of simulation experiments to estimate the influence of several parameters during periodic gaits and to compare the performance measures. Consequently, the multi-legged locomotion was simulated, in order to examine the role of the walking gait versus $\beta, L_{S}, H_{B}$ and $F_{C}$, with $V_{F}=1 \mathrm{~m} / \mathrm{s}, \quad S_{P}=1 \mathrm{~m}, \quad L_{i 1}=L_{i 2}=1 \mathrm{~m}, \quad O_{i}=0 \mathrm{~m}$, $M_{i 1}=M_{i 2}=1 \mathrm{Kg}, M_{b}=36 \mathrm{Kg}$ and $M_{i f}=0 \mathrm{Kg}$.

Due to the high number of parameters and values, in the next sub-sections we capture the optimal values by cross-relating several distinct combinations for the Wave Gait (WG).

\subsection{Step Length vs. Duty Factor}

Figure 3 depicts the three indices versus $\left(L_{S}, \beta\right)$. We conclude that $P_{a v}, D_{a v}$ and $P_{L}$ increase monotonically with $\beta$ and decrease with $L_{S}$.

\subsection{Step Length vs. Body Height}

Figure 4 shows $P_{a v}, D_{a v}$ and $P_{L}$ versus $\left(L_{S}, H_{B}\right)$. We verify that the indices decrease slightly with $H_{B}$ and $L_{S}$.

\subsection{Duty Factor vs. Foot Clearance}

Figure 5 depicts $P_{a v}\left(\beta, F_{C}\right)$ revealing that it increases with $\beta$ and $F_{C}$. Although not presented $D_{a v}\left(\beta, F_{C}\right)$ and $P_{L}\left(\beta, F_{C}\right)$ show the same type of variation with $\beta$ and $F_{C}$. This means that the robot should walk with $\beta \approx 50 \%$ and $F_{C}$ as small as possible.

The same can be concluded through Figure 6 that depicts $L_{F}\left(\beta, F_{C}\right)$. From this figure we can verify that $L_{F}$ presents a maximum for values of $\beta \approx 50 \%$ and $F_{C} \approx 0$. 

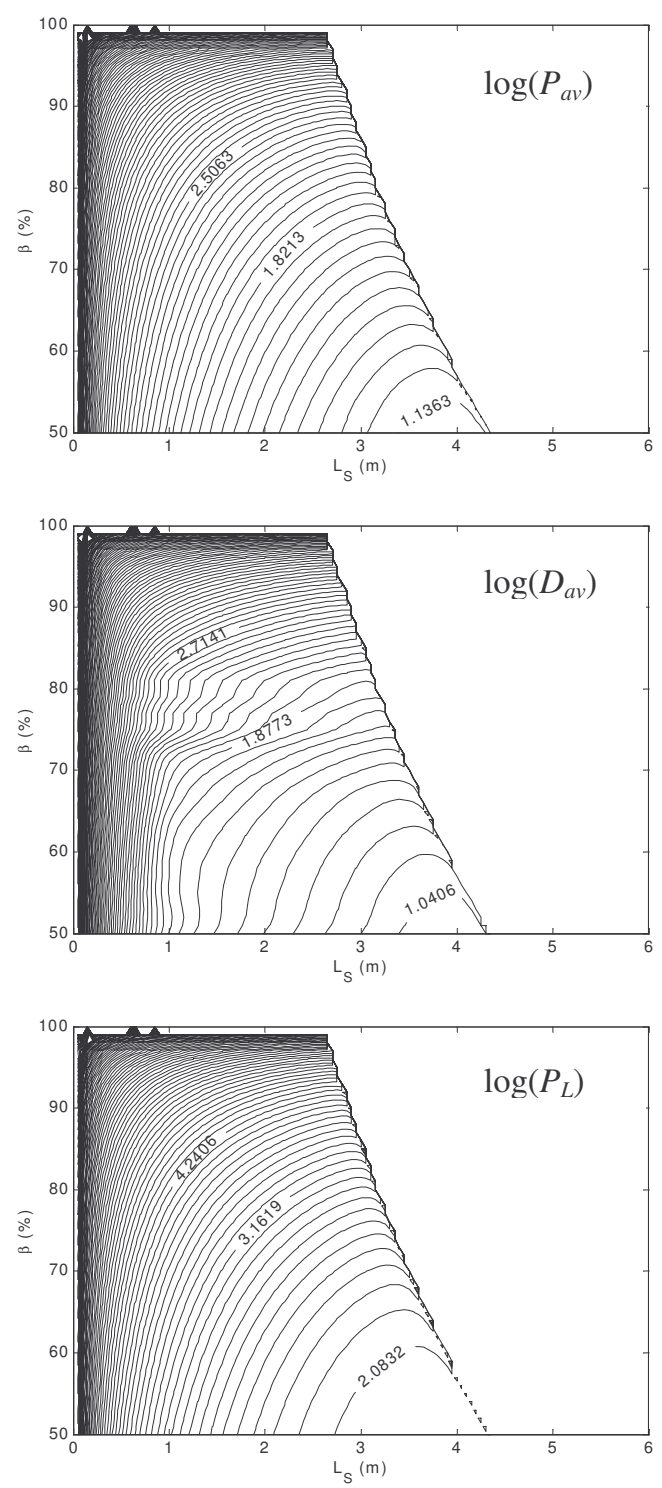

Fig. 3. Plots of $\log \left(P_{a v}\right), \log \left(D_{a v}\right)$ and $\log \left(P_{L}\right)$ vs. $\left(L_{S}, \beta\right)$ for $F_{C}=0.01 \mathrm{~m}, H_{B}=1.5 \mathrm{~m}, V_{F}=1 \mathrm{~m} / \mathrm{s}$, $W G$.

In conclusion, comparing all the previous experiments, we can establish a compromise for optimising the Wave Gait, namely that the best situation occurs for $\beta \approx 50 \%, 1.5 \leq H_{B} \leq 1.8 \mathrm{~m}$, $3.0 \leq L_{S} \leq 5.0 \mathrm{~m}$ and $F_{C}<0.1 \mathrm{~m}, \quad$ considering $V_{F}=1 \mathrm{~m} / \mathrm{s}, L_{i 1}=L_{i 2}=1 \mathrm{~m}$ and $O_{i}=0 \mathrm{~m}$.

For other periodic gaits, namely the Equal Phase Half Cycle gait $(E P H C G)$, Equal Phase Full Cycle gait $(E P F C G)$, Backward Wave gait $(B W G)$, Backward Equal Phase Half Cycle gait $(B E P H C G)$ and Backward Equal Phase Full Cycle gait $(B E P F C G)$, the performance variation with the parameters $\beta, H_{B}$, $L_{S}$ and $F_{C}$ is similar. Therefore, we need a complementary analysis (and index) in order to compare the performance of different walking gaits.

Bearing these facts in mind, we compared the total instantaneous absolute power consumption $P_{i}(t)$ (see Equation 8a) along one period of the robot walking cycle, for these periodic gaits.
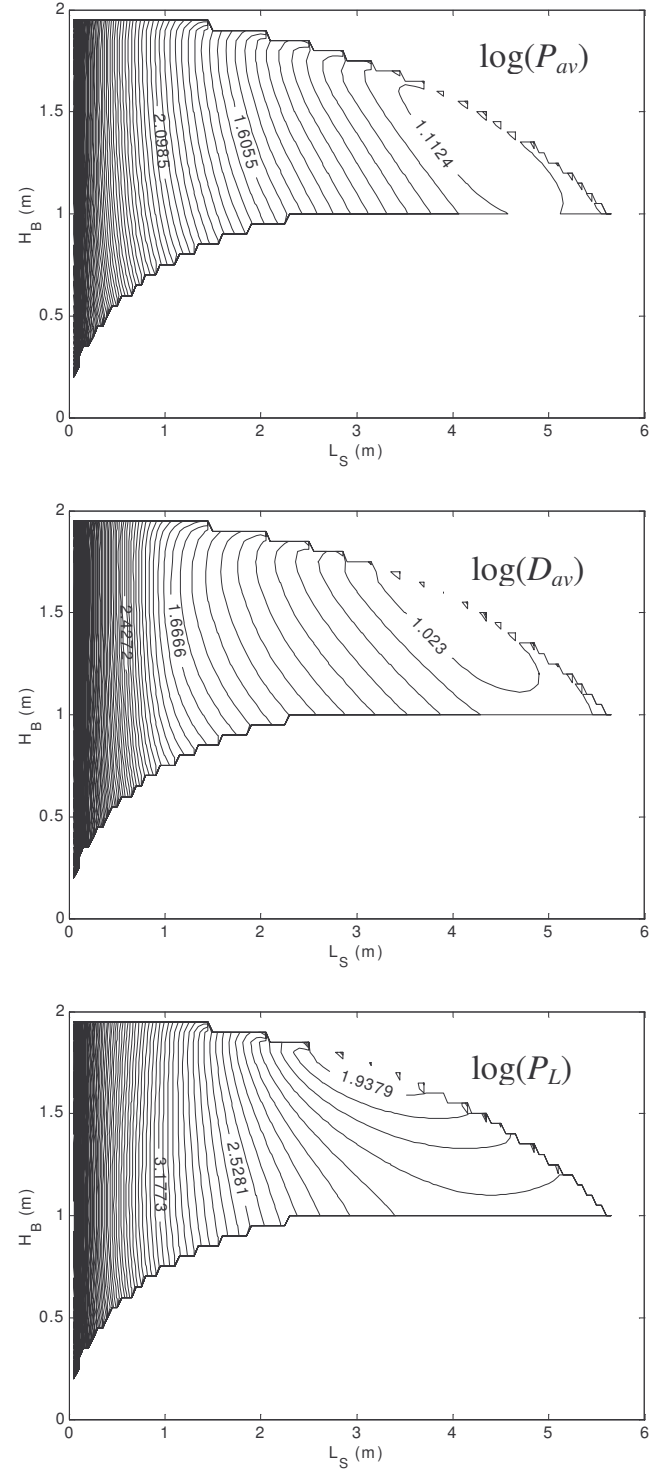

Fig. 4. Plots of $\log \left(P_{a v}\right), \log \left(D_{a v}\right)$ and $\log \left(P_{L}\right)$ vs. $\left(L_{S}, H_{B}\right)$ for $\beta=50 \%, F_{C}=0.01 \mathrm{~m}, V_{F}=1 \mathrm{~m} / \mathrm{s}$, $W G$.

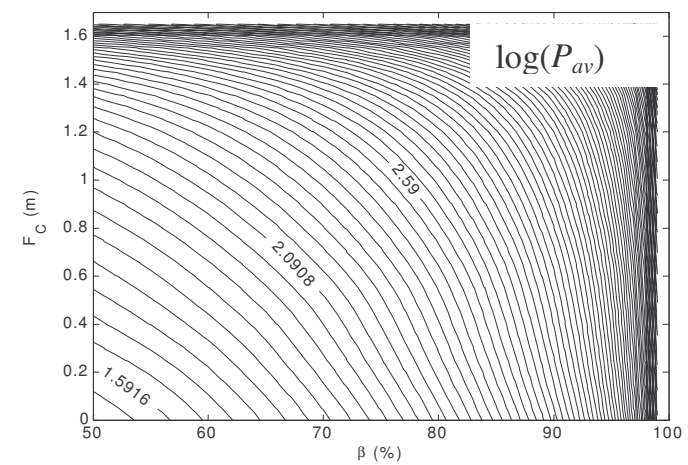

Fig. 5. Plot of $\log \left(P_{a v}\right)$, vs. $\left(\beta, F_{C}\right)$ for $L_{S}=1.9 \mathrm{~m}$, $H_{B}=1.7 \mathrm{~m}, V_{F}=1 \mathrm{~m} / \mathrm{s}, W G$.

Figure 7 shows that the $E P H C$ and $E P F C$ require $P_{i}(t)$ peaks lower than those required by the $W G$.

Since $P_{i}(t)$ for the backward gaits is similar to those of the forward gaits, we conclude that the $W G$ is less efficient than the $E P H C$ and the $E P F C$, from the viewpoint of an autonomous energy source. 


\subsection{Body Forward Velocity}

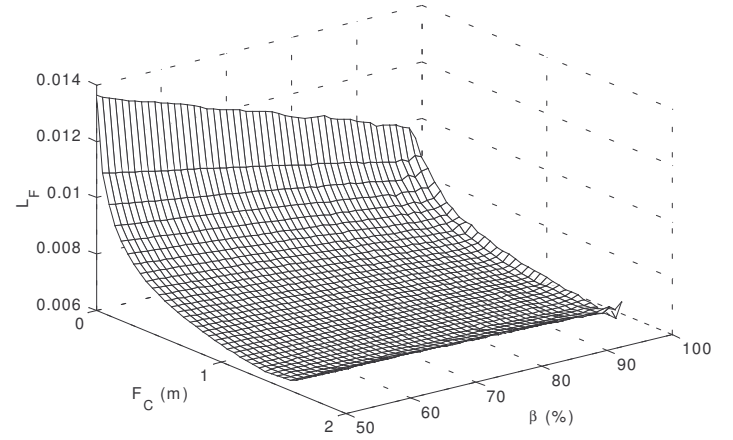

Fig. 6. Plot of $L_{F}$ vs. $\left(\beta, F_{C}\right)$ for $L_{S}=1.9 \mathrm{~m}$, $H_{B}=1.7 \mathrm{~m}, V_{F}=1 \mathrm{~m} / \mathrm{s}, W G$.
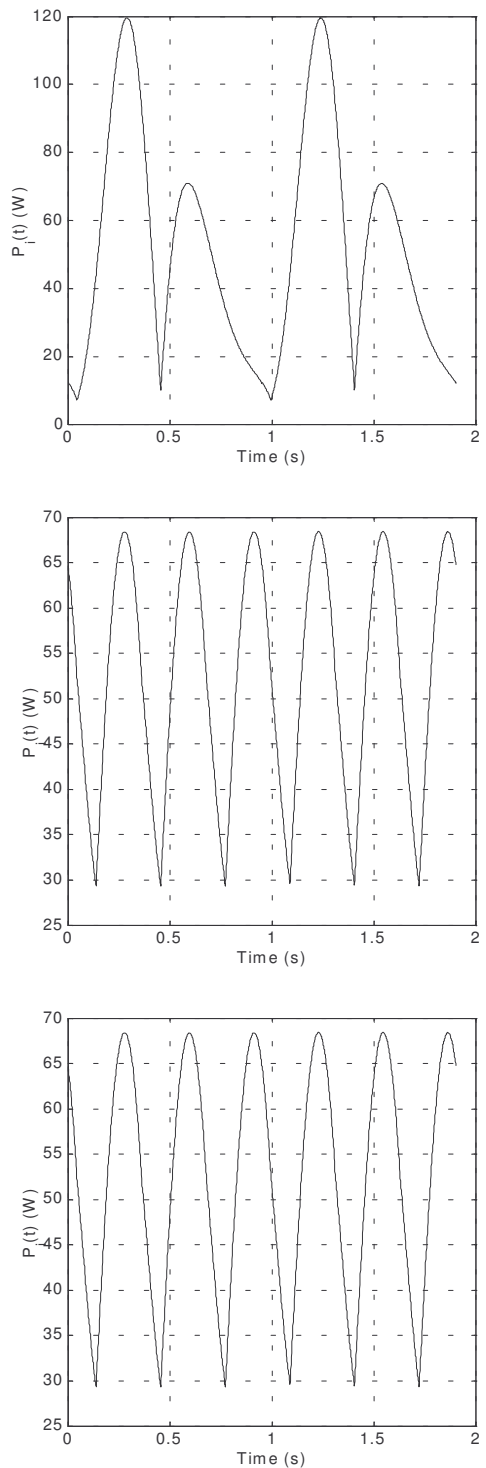

Fig. 7. Plots of $P_{i}(t)$ for $\beta=50 \%, L_{S}=1.9 \mathrm{~m}$, $H_{B}=1.7 \mathrm{~m}, \quad F_{C}=0.01 \mathrm{~m}, \quad V_{F}=1 \mathrm{~m} / \mathrm{s}, \quad W G$, EPHCG and EPFCG.

Comparing the instantaneous power for the left and right sides of the robot, we verify that all gaits show similar requirements, but the EPFCG presents lower power peaks. Moreover, in this point of view both forward and backward gaits pose similar requirements.
Figure 8 shows $\min \left[P_{a v}\left(V_{F}\right)\right]$ revealing that $P_{a v}$ increases with $V_{F}$. Furthermore, we have $P_{a v} \propto V_{F}^{1.03}$ for low velocities, while $P_{a v} \propto V_{F}^{3.02}$ for high velocities, being the "switch" between both behaviours for $V_{F} \approx 1.15 \mathrm{~m} / \mathrm{s}$. For $D_{a v}$ and $P_{L}$ we can establish similar conclusions.

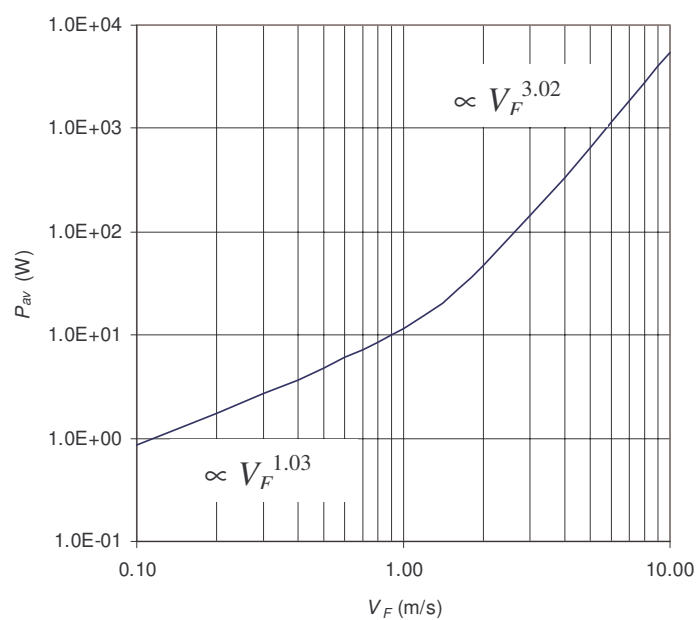

Fig. 8. Plot of $\min \left[P_{a v}\left(V_{F}\right)\right]$ for $\beta=50 \%$, $F_{C}=0.01 \mathrm{~m}, W G$.

\subsection{Foot Trajectory Offset vs. Leg Length}

In the previous experiments we considered constant link lengths and masses, namely $L_{i 1}=L_{i 2}=1 \mathrm{~m}$ and $M_{i 1}=M_{i 2}=1 \mathrm{Kg}$, for $O_{i}=0 \mathrm{~m}$. Now we study the influence of these factors upon $L_{F}, P_{a v}, D_{a v}$ and $P_{L}$. Therefore, we establish a total constant leg length and mass of $L_{t}=L_{i 1}+L_{i 2}=2 \mathrm{~m}$ and $M_{t}=M_{i 1}+M_{i 2}=2 \mathrm{Kg}$ while varying the relation between the two links, yielding $(i=1, \ldots, 6 ; j=1,2)$ $M_{i j}=\left(L_{i j} / L_{t}\right) \cdot M_{t}$.

Figure 9 shows $P_{a v}\left(O_{i}, L_{i 1}\right)$ for legs link lengths $0.2<L_{i 1}<1.7$ and hip-foot offset $-0.5<O_{i}<0.5$. We conclude that $P_{a v}$ varies slightly with $L_{i 1}$ and $O_{i}$. For values of $O_{i}$ and $L_{i 1}$ outside this interval $P_{a v}$ increases rapidly. The graphs of $\log \left(D_{a v}\right)$ and $\log \left(P_{L}\right)$ present a similar variation. From these charts we conclude that the locomotion is more efficient with $0.7<L_{i 1}<0.9 \mathrm{~m}\left(L_{i 1}+L_{i 2}=2 \mathrm{~m}\right)$ and $O_{i} \approx+0.4$ or $O_{i} \approx-0.2 \mathrm{~m}$. The same can be concluded through Figure 10 that depicts $L_{F}\left(O_{i}, L_{i 1}\right)$.

\section{CONCLUSIONS}

In this paper we have compared various dynamic aspects of multi-legged robot locomotion gaits. By implementing different motion patterns, we estimated how the robot responds to a variety of locomotion variables such as duty factor, step length, body height, maximum foot clearance, legs link lengths and foot trajectory offset. For analysing the system performance four quantitative measures were defined: the foot locomobility index, the average 

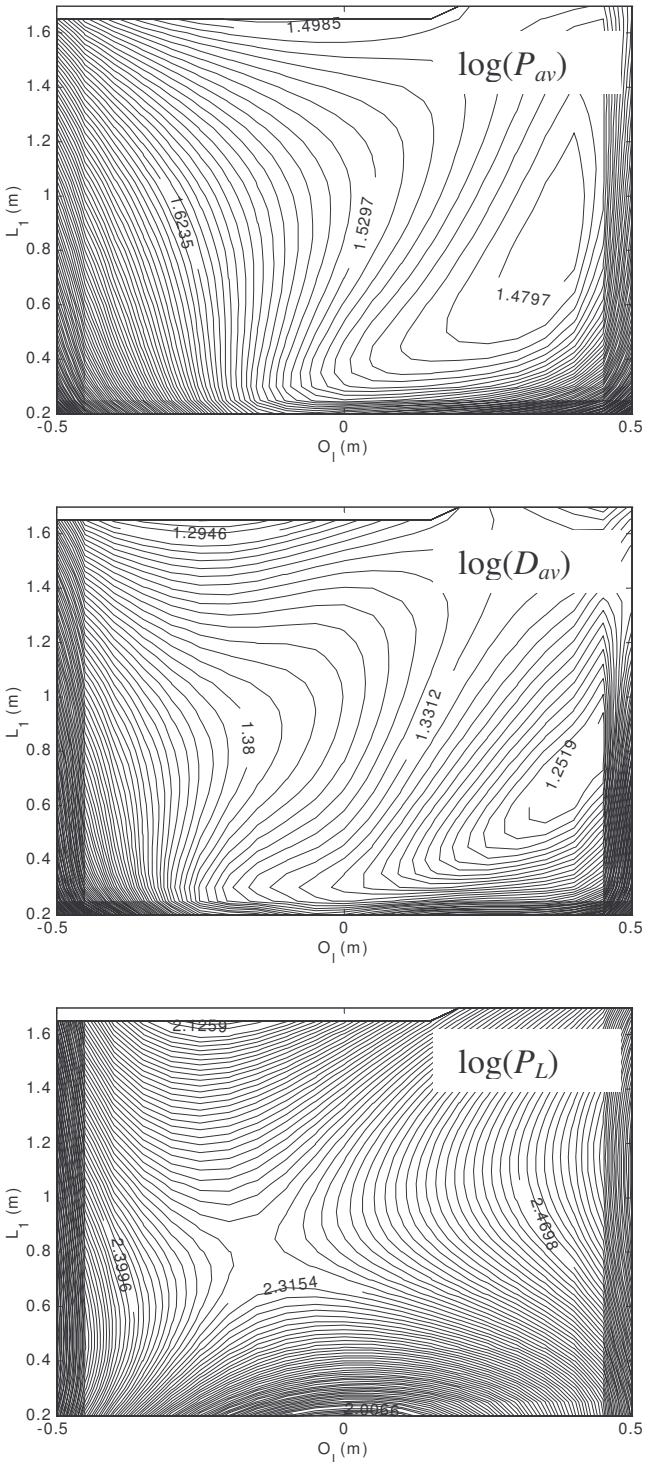

Fig. 9. Plots of $\log \left(P_{a v}\right), \log \left(D_{a v}\right)$ and $\log \left(P_{L}\right)$ vs. $\left(O_{i}, L_{i 1}\right)$ for $\beta=50 \%, L_{S}=1.8 \mathrm{~m}, F_{C}=0.01 \mathrm{~m}$, $H_{B}=1.7 \mathrm{~m}, V_{F}=1 \mathrm{~m} / \mathrm{s}, W G$.

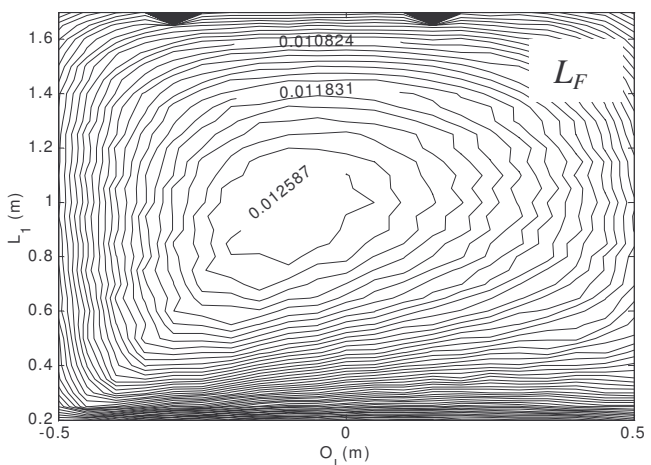

Fig. 10. Plot of $L_{F}$ vs. $\left(O_{i}, L_{i 1}\right)$ for $\beta=50 \%$, $L_{S}=1.8 \mathrm{~m}, F_{C}=0.01 \mathrm{~m}, H_{B}=1.7 \mathrm{~m}, V_{F}=1 \mathrm{~m} / \mathrm{s}$, $W G$.

power consumption, the power consumption standard deviation and the power expenditure in the actuators. Through the analysis of the results of the simulations we draw several conclusions on the best set of locomotion variables. While our focus has been on a power analysis in periodic gaits, certain aspects of locomotion are not necessarily captured by the proposed measures. Consequently, future work in this area will address the refinement of our models to incorporate more unstructured terrains, namely with distinct trajectory planning concepts. Moreover, we will also address the effects of the foot-ground interaction and a model describing the ground characteristics. The contact and reaction forces at the robot feet will enable further insight towards the development of efficient multi-legged locomotion robots.

\section{REFERENCES}

Caux, S. and Zapata, R. (1997). Modeling and Control of Biped Robot Dynamics. Robotica, 17, 413-426.

Gregorio, P., Ahmadi, M. and Buehler, M. (1997). Design, Control, and Energetics of an Electrically Actuated Legged Robot. IEEE Trans. on Sys., Man and Cyber., 27, n. 4.

Jiménez, M. A. and Santos, P. G. (1997). TerrainAdaptive Gait for Walking Machines. The Int. J. of Robotics Research, 16, 320-339.

Lapshin, V. V. (1995). Energy Consumption of a Walking Machine. Model Estimations and Optimization. IEEE Int. Conf. on Advanced Robotics, Hungary, pp. 420-425.

Manko, D. J. (1992). A General Model of Legged Locomotion on Natural Terrain. Kluwer, Westinghouse Electric Corporation.

Nelson, G. M. and Quinn, R. D. (1999). Posture Control of a Cockroach-like Robot. IEEE Control Systems, 19, 9-14.

Pfeiffer, F., Eltze, J. and Weidemann, H.-J. (1995). The TUM Walking Machine. Intelligent Automation and Soft Computing, 1, 307-323.

Silva, F. M. and Machado, J. A. T. (1999). Energy Analysis During Biped Walking. IEEE Int. Conf. on Robotics and Automation, USA.

Silva, M. F., Machado, J. A. T. and Lopes, A. M. (2001). Performance Analysis of Periodic Gaits in Multi-Legged Locomotion. IEEE Int. Conf. on Advanced Robotics, Hungary.

Silva, M. F., Machado, J. A. T. and Lopes, A. M. (2001). Energy Analysis of Multi-Legged Locomotion Systems. $4^{\text {th }}$ Int. Conf. on Climbing and Walking Robots, Germany.

Song, S.-M. and Waldron, K.J. (1989). Machines that Walk: The Adaptive Suspension Vehicle. The MIT Press.

Venkataraman, S. T. (1996). A Model of Legged Locomotion Gaits. IEEE Int. Conf. on Robotics and Automation, USA.

Wettergreen, D., Pangels, H. and Bares, J. (1995). Behavior-based Gait Execution for the Dante II Walking Robot. IEEE Int. Conf. on Intelligent Robots and Systems, USA.

Wettergreen, D. and Thorpe, C. (1992). Gait Generation for Legged Robots. IEEE Int. Conf. on Intelligent Robots and Systems.

Yoshikawa, T. (1990). Foundations of Robotics Analysis and Control. The MIT Press.

Zielinska, T. (2000). Efficiency Analysis in the Design of Walking Machines. J. of Theoretical and Applied Mechanics, 38, n. 3, 693-705. 